\title{
Contradiction as a Form of Contractual Incompleteness*
}

\author{
Dana Heller ${ }^{\dagger}$ and Ran Spiegler ${ }^{\dagger}$ \\ First version: December 2005 \\ This version: September 2006
}

\begin{abstract}
A simple model is presented, in which contradictory instructions are viewed as a type of contract incompleteness. The model provides a complexity-based rationale for contradictory instructions. If there are complexity bounds on the contract, there may be an incentive to introduce contradictions, leaving for another agent the task of interpreting them. The optimal amount of contradictions depends on the complexity bound, the conflict of interests with the interpreter, and the institutional constraints on his interpretations. In particular, a higher complexity bound may result in a larger amount of contradictions.
\end{abstract}

${ }^{*}$ We are grateful to an editor and a referee for useful comments.

$\dagger$ National Economics Research Associates Inc. E-mail: dana.heller@nera.com.

${ }^{\ddagger}$ School of Economics, Tel Aviv University. E-mail: rani@post.tau.ac.il. URL: http://www.tau.ac.il/ rani. 


\section{Introduction}

The contract-theoretic literature has identified two different notions of contract incompleteness. First, a contract is incomplete if it is not as fine as it should be, given verifiability constraints. Second, a contract is incomplete if it has lacunae - i.e., it ignores some states of the world. In this paper we identify a third type of contract incompleteness: contradictory instructions. To motivate this idea, consider a contract that consists of two clauses: (i) in case of an earthquake, take action $a$; (ii) in case of a fire, do not take action $a$. This contract has a lacuna, as it fails to specify what happens when neither of the two events (earthquake, fire) takes place. However, the contract also contains a contradiction: if both events happen simultaneously, the contract specifies mutually contradictory actions.

The contradiction is due to the fact that the contract is a function that assigns actions to events, rather than mutually exclusive states. Existing contract-theoretic literature typically formalizes a contract as a function from states to actions (a couple of exceptions are Battigalli and Maggi (2002) and Shavell (2006); see below). Such a formalism can capture the familiar types of contract incompleteness, but it cannot capture contradictions. However, real-life contracts are written in natural language. A contingency stated in natural language typically corresponds to an event, rather than to an individual state. Therefore, it may be more fitting to formalize a contract as a function from events to actions. Such a formalism can accommodate contradictions.

In this short paper we study a simple model that provides a rationale for this third type of contract incompleteness, based on the notion of contracts as functions from events to actions. The rationale is a variant on the writing-complexity argument for contract incompleteness, due to Dye (1985), Anderlini and Felli (1994,1999) and Battigalli and Maggi (2002). In

the model, one agent, referred to as the Writer, unilaterally writes down a list of instructions for another agent, referred to as the Interpreter, to carry 
out. There is a conflict of interests between the Writer and the Interpreter. The state space is $[0,1)$. An instruction consists of an event - i.e., a subset of $[0,1)$ - and an action. Two instructions are contradictory if they specify intersecting events and different actions. A lacuna exists if there are states of the world which are not covered by any instruction.

When a state of nature is covered by exactly one instruction, the Interpreter is obliged to take the action that this instruction specifies in this state. However, when the state falls into a lacuna or a contradiction, the Interpreter exercises discretion. We impose a single constraint on the Interpreter's discretion: if two states belong to the same set of events in the Writer's instructions list, then the Interpreter must take the same action in both states.

The following scenario illustrates the model. Suppose that the Writer is a set of contracting parties or a legislature, whereas the Interpreter is a court of law. An "instructions list" is a contract or a law. When a state is realized and gives rise to a lacuna or a contradiction, the court steps in and provides an interpretation. The constraint we impose on the court's discretion reflects a precedent system. Even if the court can observe the true state, a precedent system implies that the court must arrive at the same ruling in two states which are equivalent in terms of the contract or law in question.

If there were no limitations on the Writer's instructions list, he would want to write down a complete contract and leave no discretion to the Interpreter, because of their conflict of interests. However, we assume that there are two complexity bounds on the Writer's instructions list. First, there is an upper bound $K$ on the number of instructions on the list. Second, the family of events on which he can draw is restricted. Specifically, an admissible event is a union of no more than $r$ disjoint intervals in $[0,1)$. Thus, $r$ is a measure of the richness of the language in which events are phrased.

Complexity measures are inevitably artificial, and the present case is no exception. Nevertheless, we believe that it is reasonable to interpret $K$ and $r$ 
as measures of the complexity of writing the instructions list. The larger the number of instructions, and the larger the number of contingencies on which each instruction conditions, the more costly it is to write the instructions list. ${ }^{1}$ As Battigalli and Maggi (2002) state:

"...we have in mind costs that are, broadly speaking, proportional to the amount of detail in the contract, such as the cost of figuring out the relevant contingencies and obligations, the cost of thinking how to describe them, the cost of time needed to write the contract, and the cost of lawyers." (Battigalli and Maggi (2002), p. 798)

Thus, a key feature of our model is that the Writer's decision how much interpretational freedom to assign the court is strategic, and takes writing costs into account. We believe that this is a realistic feature of the contracting or legislative process. To quote Shavell (2006):

"We also observe that the courts actively engage in the interpretation of contracts. The courts fill gaps in contracts, resolve conflicts and ambiguities of language, and sometimes replace the parties' express terms with the courts' terms ... Moreover, the interpretation of contracts is widely understood to influence how parties write contracts: the more closely the courts' interpreted contracts resemble the parties' true wishes, the more willing the parties are to leave gaps and to write fairly general terms, whereas parties are more willing to take extra pains to write more detailed contracts when courts refrain from interpreting terms or interpret

\footnotetext{
${ }^{1}$ Note that we capture writing complexity by placing the upper bounds $K$ and $r$, rather than by specifying a cost function which is increasing in $K$ and $r$. This is done to facilitate exposition, and does not affect the qualitative results.
} 
terms in ways that run counter to their true desires." (Shavell (2006), p. 290)

The model studied by Shavell (2006) shares our model's general structure, the crucial difference being that it rules out contradictions. Nevertheless, as the above quote attests, Shavell acknowledges the relevance of contradictions for the act of interpretation. Indeed, according to Farnsworth (1999), a substantial proportion of contract trial cases in the American judicial system are concerned with interpretation, which usually involves overriding terms, ambiguities of language, and internal contradictions in terms.

Given the complexity constraints, the Writer has an incentive to create contradictions because they refine the effective partition that is induced by the instructions list. Recall the earthquake-fire example. There are four states of the world: nothing happens, only an earthquake occurs, only a fire occurs, both an earthquake and a fire occur. A complete contract specifying different actions for different states would require at least four instructions. However, if there is no conflict of interests between the two agents, the twoclause contract achieves the same outcome. By allowing the Interpreter to "interpret" contradictions and lacunae, we effectively refine the instructions list. However, when there is a conflict of interests between the two agents, the Writer has to trade off this consideration with his desire not to give the Interpreter too much discretion.

Using a standard Crawford-Sobel model of the conflict of interests between the Writer and the Interpreter, we show that the equilibrium degree of vagueness - i.e., the measure of states that fall into contradictory instructions or lacunae in subgame perfect equilibrium - is increasing with $r$. Such a clear-cut relation does not exist with respect to $K$. When $r=1$, the equilibrium degree of vagueness decreases with $K$. In contrast, when $r$ is very large, an increase in $K$ may result in a greater degree of vagueness. Nevertheless, if $K$ is sufficiently large, there no lacunae or contradictions in equilibrium. 
These results may be surprising at first glance: one would expect a priori that a Writer with more "linguistic resources" would leave less for the Interpreter to interpret, due to their conflict of interests. The intuition for our results is that with larger linguistic resources it is possible to generate a lot more contradictions, thus enabling the Interpreter to refine his strategy. Because the Writer is risk-averse, this strengthens his incentive to introduce contradictions.

The characterization of the equilibrium instructions list is of particular interest in the case of $r=1$ - i.e., when the events on which instructions condition are intervals. The $K$ events on the list constitute a pseudo-interval partition with "fuzzy borders". This structure has a natural interpretation: the Writer invokes categories such as "very low", "moderately low", "moderately high", etc., but the exact demarcation of these categories is fuzzy.

The rationale for contradictions relies on the way the Interpreter is allowed to interpret contradictions. We demonstrate this point by examining two alternative "methods of interpretation": (i) when a state is covered by several contradictory instructions, the Interpreter is bound to select one of the actions specified in these instructions; (ii) there are no constraints at all on the Interpreter's discretion in case of lacunae or contradictions. In both cases, the rationale for contradictions disappears. These observations demonstrate the importance that norms of contract interpretation have for contractual incompleteness, and consequently the need for continued exploration of the ways in which courts interpret incomplete contracts.

\section{Related literature}

Dye (1985) is the first paper to model explicitly the writing costs of contracts. His measure of a contract's complexity is the number of contingencies on which the contract conditions (this is analogous to $K$ in the present paper). However, he assumes that the contract specifies a partition of the state space, thereby ruling out both lacunae and contradictions.

Battigalli and Maggi (2002) propose to model contracts as functions of 
events. They analyze a propositional model of contracts, in which events are defined by disjunctions and conjunctions of elementary propositions and their negations. However, they exclude contradictions, by focusing on what they call "feasible" contracts. ${ }^{2}$ Our paper can be viewed as a modest contribution to their line of thought.

Shavell (2006) is the closest work to the present paper. Shavell models a contract as a function of events, yet like Battigalli and Maggi (2002), he rules out contradiction by assuming that these events are mutually exclusive. Shavell assumes that contracts are interpreted by the court, and studies the contracting parties' optimal choice of contracts, given the court's method of interpretation. Thus, our paper may be viewed as an extension of Shavell's model in the direction of incorporating contradictions into contracts.

Posner (2005) provides a reduced-form, cost-benefit analysis of contract interpretation, weighing the negotiation and interpretation costs associated with a contract. Anderlini, Felli and Postlewaite $(2001,2003)$ are the only other papers that we are aware of, in which the court plays an active part in a contract-theoretic model. In their model, the court can void the contract in case of lacunae (which arise from unforeseen contingencies). When a court voids the contract, the contracting parties can renegotiate. The model does not accommodate contradictions. These papers study the effects of the court's policy on the contracting parties' incentives.

As Lipman (2003) points out, there is a close link between the study of contradictions in contracts and the study of vagueness in natural language. Hopefully, further exploration of the former might also shed light on the latter.

\footnotetext{
${ }^{2}$ Battigalli-Maggi acknowledge the possibility of contradictions (see footnote 11 in their paper), and the court's need to intervene in such cases, but they do not develop this idea.
} 


\section{The model}

Two agents, a Writer and an Interpreter, play a sequential-move game. Their underlying conflict of interests is modeled as in the well-known example due to Crawford and Sobel (1982). Let $X=[0,1)$ be a set of states of nature. A subset of $X$ is called an event. The state is drawn from the uniform probability measure $\mu$ over $[0,1)$. The set of actions which are available to the Interpreter is the set of real numbers $\mathbb{R}$. Player $j$ 's vNM utility function is given by $u_{j}(x, a)=-\left(x-m_{j}+a\right)^{2}$, where $m_{j} \in \mathbb{R}$ is his "ideal point". Without loss of generality, let $m_{\text {writer }}=0$ and denote $m_{\text {interpreter }}=\mathrm{m}$. Assume that $m \neq 0$.

The order of moves is as follows. The Writer moves first. He chooses an instructions list, which is a sequence $c=\left(e_{k}^{c}, a_{k}^{c}\right)_{k=1, \ldots, K}$, where $e_{k}^{c}$ is an event and $a_{k}^{c}$ is an action. We refer to $c_{k}=\left(e_{k}^{c}, a_{k}^{c}\right)$ as the $k$ th instruction on the list $c$. Let $E^{c}=\left\{e_{1}, \ldots, e_{K}\right\}$. The set $E^{c}$ need not be a partition of $X$. We assume that for every $k, e_{k}^{c} \in \mathcal{E}$, where $\mathcal{E}$ is a family of admissible events.

We introduce $\mathcal{E}$ in order to capture bounds on the complexity, or richness, of the language for describing events. Therefore, we impose some structure on $\mathcal{E}$. Given that the state space is $[0,1)$, a natural "primitive" event is an interval. Thus, we assume that every element in $\mathcal{E}$ is a union of no more than $r \geq 1$ disjoint intervals in $[0,1)$. The larger $r$, the richer the language in which events are phrased. ${ }^{3}$

Before we describe the Interpreter's move, let us introduce some notation. For every $J \subseteq\{1, . ., K\}$, define

$$
p_{J}^{c}=\left\{x \in[0,1) \text { s.t. } x \in e_{k}^{c} \forall k \in J \text { and } x \notin e_{k}^{c} \forall k \notin J\right\}
$$

The set $p_{J}^{c}$ consists of all states which are covered by the subset of instructions $J$ in the instructions list $c$. Note that the collection $\left\{p_{J}^{c}\right\}_{J \subseteq\{1, . ., K\}}$ is a partition

\footnotetext{
${ }^{3}$ The intervals may be open or closed. This distinction is immaterial because of the non-atomicity of $\mu$. For notational consistency, we assume that the intervals are half-open of the form $\left[x_{l}, x_{h}\right)$.
} 
of $X$ : two states belong to the same $p_{J}^{c}$ if and only if they are covered by exactly the same subset $J$ of instructions. We refer to the collection of non-empty sets in $\left\{p_{J}^{c}\right\}_{J \subseteq\{1, ., K\}}$ as the effective partition induced by $E^{c}$, and denote it by $P^{c}$. For every $x \in[0,1)$, let $J^{c}(x)$ denote the subset of instructions that cover $x$. Formally, $J^{c}(x)=\left\{k \in\{1, \ldots, K\}\right.$ s.t. $\left.x \in e_{k}^{c}\right\}$.

Whenever $\left|J^{c}(x)\right|=1, x$ is covered by exactly one instruction - hence, there is no vagueness regarding the action that the Interpreter needs to take in state $x$. When $\left|J^{c}(x)\right|=0, x$ falls into a lacuna, because it is not covered by any instruction. When $\left|J^{c}(x)\right|>1, x$ is covered by multiple instructions. If the actions specified by these instructions are different, the instructions are mutually contradictory. Given an instructions list $c$, let $\delta(c)$ be the amount of vagueness that characterizes it. Formally, $\delta(c)$ is the $\mu$-measure of states $x$ for which $\left|J^{c}(x)\right| \neq 1$.

The Interpreter moves after the Writer has made his choice. Given $\left(e_{k}^{c}, a_{k}^{c}\right)_{k=1, \ldots, K}$, the Interpreter chooses a function $I: P^{c} \rightarrow \mathbb{R}$. That is, the Interpreter's strategy is measurable with respect to the effective partition induced by $E^{c}$. We impose a single condition on $I$ :

Condition 1 For every state $x$, if $J^{c}(x) \neq \varnothing$ and $a_{k}^{c}=a$ for every $k \in$ $J^{c}(x)$, then $I\left(p_{J}^{c}\right)=a$.

In particular, if $J$ is a singleton $\{k\}$, then $I\left(p_{J}^{c}\right)=a_{k}^{c}$. That is, the Interpreter's strategy must coincide with the Writer's strategy when $p_{J}^{c}$ does not represent a lacuna or a contradiction. In other words, the Interpreter is allowed to exercise discretion only in case of a lacunae or a contradiction.

The model captures situations in which one agent writes down a set of instructions to be carried out by another agent. The first agent can build contradictions or lacunae into his instructions. On one hand, the contradictions and lacunae give the second agent discretion, which runs against the first agent's interests. On the other hand, they help the second agent refine 
his strategy, which is favorable to the first agent because it reduces decision errors.

We interpret an instructions list as a law or a contract. The Writer represents a set of contracting parties, or a legislature. Accordingly, we may view the Interpreter as a court of law. The court is asked to interpret contracts and laws when those contain contradictions and lacunae. Under this interpretation, the assumption that $I$ is measurable with respect to $P^{c}$ reflects a "precedent system". Even if the court knows the state of nature, the precedent system forces the court to make the same decision in two states which are indistinguishable in the eyes of the law.

The parameters $K$ and $r$ are indicators of the amount of detail in the law: $K$ is the number of contingencies on which actions are conditioned, and $r$ measures the complexity of each contingency. Economists are sometimes suspicious of the notion of writing costs, as if it involved the mere cost of ink. However, contracts and laws that contain a greater number of complex contingencies are more costly to prepare, because the act of putting the instructions into words is difficult and time consuming.

We conclude this section with a number of comments regarding the interpretation of the model.

Lacunae versus contradictions. The distinction between lacunae and contradictions in our model is quite artificial. A lacuna is simply identified as a particular cell $p_{\varnothing}^{c}$ in the effective partition, and treated just like any other cell $p_{J}^{c}$ for which $|J|>1$. We could modify our model by allowing the Writer to add a "basket clause" to the instructions list, which assigns a particular action to any $x$ which belongs to none of the events in the list. The motivation for such a modification is that the writing costs associated with a basket clause are negligible, compared to the costs associated with the other instructions in the list. However, this modified model would be more cumbersome to analyze than ours, while the qualitative results would be the same. 
Alternative complexity measures. The basic building blocks in the description of events are intervals. Alternatively, we could assume that a primitive event is an inequality $\left(x>x_{0}\right.$ or $\left.x<x_{0}\right)$, so that an event in $\mathcal{E}$ would be a combination of unions or intersections of inequalities. Since an interval is no more than an intersection of two inequalities (except for intervals of the form $\left(0, x_{0}\right)$ or $\left(x_{0}, 1\right)$, which can be described as a single inequality), our analysis would be qualitatively the same. Another notion of complexity, employed by Anderlini and Felli (1994) is based on the concept of computable functions. The distinction between computable and incomputable contracts is irrelevant in the present context. As we shall see below, when $K$ is sufficiently large, the Writer's incentive to create lacunae and contradictions disappears. Thus, contract incompleteness in the sense we focus on does not rely on this distinction, but on the simpler question of the contract's length.

Further constraints on interpretation. We place no restriction on the Interpreter's discretion in case of lacunae or contradictions, apart from the measurability condition which captures a precedent system. One could imagine additional constraints. For instance, suppose that when two instructions $k$ and $j$ specify contradictory actions, $a_{k}^{c}$ and $a_{j}^{c}$, for a certain state, the Interpreter's choice of action must reflect some "averaging" of the two actions. That is, the Interpreter cannot totally disregard the actions specified by the conflicting instructions. Such additional constraints on the Interpreter's strategy are assumed away, for the sake of expositional simplicity. In Section 4 , we discuss alternative "methods of interpretation".

Costs of interpretation. One could argue that while focusing on writing costs, we completely ignore the cost of interpreting contradictions and lacunae (see Posner (2005) for a discussion of such costs). If the Writer does not bear the interpretation costs, including them will not affect our analysis. Of course, if the Writer does take into account the costs of interpretation, we need a more elaborate model to analyze the trade-off between writing costs and interpretation costs. 


\section{Analysis}

In this section we analyze the subgame perfect equilibrium in the game. In particular, we are interested in the equilibrium degree of vagueness $\delta\left(c^{*}\right)$ i.e., the measure of states that fall into lacunae and contradictions - which is induced by the equilibrium instructions list $c^{*}=\left(e_{k}^{*}, a_{k}^{*}\right)_{k=1, \ldots, K}$. Denote $E^{*}=$ $\left\{e_{1}^{*},,, ., e_{K}^{*}\right\}$, and let $P^{*}=\left\{p_{J}^{*}\right\}_{J \subseteq\{1, \ldots, K\}}$ be the induced effective partition.

The players' quadratic utility function has an immediate implication, which is well-known and therefore not proved here.

Proposition 1 For any set $B \subset[0,1)$, let $a_{j}(B) \equiv \arg \max _{a} E_{B} u_{j}(x+a)$. Then, $E_{B}\left[x+a_{j}(B)\right]=m_{j}$.

That is, if the Interpreter has discretion over some event $B$, he will choose the action that adjusts the expected value of $x+a$ to be $m$. If the Interpreter lacks discretion over $B$, the Writer will choose the action that adjusts the expected value of $x+a$ to be 0 .

This observation greatly facilitates our analysis. In the effective partition $P^{*}$, every set $p_{J}^{*}$ for which $|J| \neq 1$ is associated with a probability distribution over $x+a$, whose mean is $m$. Similarly, every set $p_{J}^{*}$ for which $|J|=1$ is associated with a probability distribution over $x+a$, whose mean is 0 . The Writer faces the following trade-off. If he creates contradictions, he refines the effective partition. On one hand, the refinement reduces the variance of the probability distribution over $x+a$. On the other hand, the refinement delegates more events to the Interpreter, thereby moving the expected value of $x+a$ away from the Writer's ideal point.

Let us turn to the structure of contradictions and lacunae. The number of contradictions induced by the equilibrium instructions list is the number of sets $p_{J}^{*}$ in $P^{*}$ for which $|J|>1$. We will say that $E^{*}$ contains a lacuna if $p_{\varnothing}^{*}$ is non-empty. 
Proposition 2 Suppose that $\delta\left(c^{*}\right)>0$. Then:

(i) $P^{*}$ is an intervals partition.

(ii) The number of contradictions induced by $c^{*}$ is the maximal possible, given $K$ and $r$.

(iii) $E^{*}$ contains a lacuna.

(iv) All cells $p_{J}^{*}$ with $|J|=1$ have the same measure, and all cells $p_{J}^{*}$ with $|J| \neq 1$ have the same measure.

Proof. Let us prove part (i) first. Let $c$ be an instructions list whose induced effective partition $P^{c}$ is not an intervals partition. Our first step is to show that $c$ can be transformed into another instructions list, whose induced effective partition constitutes an intervals partition and contains as many cells as $P^{c}$. By assumption, i.e., one of the elements $p_{J}^{c}$ in $E^{c}$ is a union of $n>1$ disjoint intervals. Let us try to transform $c$ so that one of these intervals $\left[x_{1}, x_{2}\right)$ is subtracted from $p_{J}^{c}$. In order to do so, we need to select some instruction $k \in J$ and subtract $\left[x_{1}, x_{2}\right)$ from $e_{k}^{c}$. Since $n>1$, the modified $p_{J}^{c}$ remains non-empty.

This modification is infeasible only if it turns $e_{k}^{c}$ into a union of more than $r$ disjoint intervals. But this can occur only if an adjacent interval (say, $\left[x_{2}, x_{3}\right)$, w.l.o.g) belongs to $p_{L}^{c}$ for some $L \supset J$. In this case, for every $l \in L \backslash J$, we can modify $e_{l}^{c}$ by lengthening one of its intervals so that it contains $\left[x_{1}, x_{2}\right)$. Note that this additional modification merely expands one of the intervals of $p_{L}^{c}$, without changing the number of disjoint intervals of which it consists. In this fashion we manage to induce an effective partition which differs from $P^{c}$ in that the interval $\left[x_{1}, x_{2}\right)$ is subtracted from $p_{J}^{c}$, without reducing the number of cells in the effective partition. We can then reiterate this type of modification, until we get an effective partition which is an intervals partition and contains as many cells as $P^{c}$.

To complete the proof of part (i), we need to show that it is optimal for the Writer to construct an instructions list which induces an effective partition satisfying two properties: first, it is an intervals partition; and 
second, it contains the largest number of cells that is feasible under $(K, r)$. By Proposition 1, each of the $K$ cells $p_{J}^{*}$ in $P^{*}$ with $|J|=1$ induces a probability distribution over $x+a$, whose mean is 0 . Similarly, each cell $p_{J}^{*}$ in $P^{*}$ with $|J| \neq 1$ induces a probability distribution over $x+a$, whose mean is $m$. The Writer's vNM utility function is quadratic. Therefore, conditional on a delegated event $B$, and given $\mu(B)$, the Writer's expected utility is higher if $B$ is an interval. Similarly, given that $B$ is an interval, the Writer's expected utility conditional on $B$ is higher if $\mu(B)$ is lower. Therefore, if the largest effective partition given $(K, r)$ is implementable as an intervals partition, the Writer will find it optimal. Moreover, because the Writer is risk-averse, it is optimal for him that all intervals $p_{J}^{*}$ with $|J|=1$ have the same measure, and all intervals $p_{J}^{*}$ with $|J| \neq 1$ have the same measure. Obviously, the largest possible effective partition contains the largest possible number of contradictions and a lacuna. This also proves parts (ii)-(iv).

The intuition for this result is simple. Suppose that $E^{*}$ is not a partition - i.e., it delegates a non-empty set of states $B$ to the Interpreter. By Proposition 1 , every event that is delegated to the Interpreter induces a probability distribution over $x+a$ with expected value $m$. Because the Writer is riskaverse, it is optimal for him to split $B$ into as many intervals as possible, thereby reducing the variance of this distribution. This is attained by creating a lacuna and as many contradictions as possible. The less trivial part of the proof is to show that it is in fact possible to induce an effective partition which both contains the largest number of cells that is feasible given $(K, r)$, and constitutes an intervals partition.

In the remainder of this section, we analyze the cases of $r=1$ and $r>1$ separately. 


\subsection{The case of $r=1$}

This special case is of particular interest, for two reasons. First, the family of admissible events is intuitively the simplest possible, given the state space. Second, the equilibrium instructions list turns out to have an interesting structure. We will say that $c$ induces a fuzzy partition if there exists a numbering of the elements in $E$, such that: (i) the only allowed intersections between $e_{k}^{c}$ and $e_{j}^{c}$ are as follows: $k$ and $j$ must be consecutive, and neither interval contains the other; (ii) the lacuna is either $\left[0, x^{*}\right)$ or $\left[x^{*}, 1\right)$.

In a fuzzy partition, adjacent events are "almost" disjoint, except that the border between them is blurred. Thus, the effective partition consists of $2 K$ intervals, of which $K$ intervals are delegated to the Interpreter.

Proposition 3 The equilibrium instructions list $c^{*}$ induces a fuzzy partition. All undelegated intervals in $P^{*}$ are of equal measure $\left(1-\delta\left(c^{*}\right)\right) / K$, and all delegated intervals in $P^{*}$ are of equal measure $\delta\left(c^{*}\right) / K$.

Proof. By Proposition 2, the effective partition induced by $c^{*}$ is an intervals partition. No effective partition can contain more than $K$ undelegated intervals. A fuzzy partition contains exactly $K$ undelegated intervals, as well as $K$ delegated intervals. Therefore, all we need to show is that no effective partition can contain more than $2 K$ intervals. To see why this must be true, note that since $r=1$, every feasible $c$ satisfies $e_{k}^{c}=\left[x_{k}^{l}, x_{k}^{h}\right]$ for all $k=1, \ldots, K$. Therefore, the effective partition is an intervals partition, in which two adjacent intervals are demarcated by some $x_{k}^{l}$ or $x_{k}^{h}$. Therefore, the effective partition cannot contain more than $2 K$ intervals. It follows that a fuzzy partition is optimal for the Writer. The measure of each delegated and undelegated cell in the fuzzy partition follows directly from part (iv) of Proposition 2. 
Proposition 4 The equilibrium amount of vagueness is

$$
\delta\left(c^{*}\right)=\max \left[\frac{1}{2}-2 K^{2} m^{2}, 0\right]
$$

Proof. By Proposition 3, the Writer's expected utility conditional on a delegated interval is $-\left(m^{2}+\delta^{2} / 12 K^{2}\right)$, and his expected utility conditional on an undelegated interval is $-(1-\delta)^{2} / 12 K^{2}$. Therefore, $\delta\left(c^{*}\right)$ is the solution to the following problem:

$$
\min _{\delta} \delta \cdot\left[m^{2}+\frac{\delta^{2}}{12 K^{2}}\right]+(1-\delta) \cdot \frac{(1-\delta)^{2}}{12 k^{2}}
$$

which yields the desired solution.

The interpretation of this pair of results is attractive, in the sense that it is suggestive of features discernible in real-life laws and contracts. The Writer chooses the events in the instructions list so that they constitute a pseudo-partition with fuzzy borders. It is as if the language that the Writer employs has a more-or-less clear notion of the meaning of the words "very bad", "bad", "moderately good", etc., but their demarcation is fuzzy. For any instruction $\left(e_{k}^{*}, a_{k}^{*}\right)$ in the optimal list, the action $a_{k}^{*}$ is targeted at the set of "clear-cut" cases, in which the realized state is unquestionably covered by instruction $k$ only. The ambiguous "borderline" cases are left for the Interpreter to decide.

The comparative statics are also intuitive. As $K$ decreases (capturing a situation with high writing costs), and as the conflict of interests between the two parties diminishes, the ambiguous borderline cases become more prevalent. If $K m>\frac{1}{2}$, there is no delegation at all in equilibrium.

\subsection{The case of $r>1$}

What happens to the equilibrium degree of vagueness when we raise $r$, thus enriching the Writer's language? 
Proposition $5 \delta\left(c^{*}\right)$ weakly increases with $r$.

Proof. By Proposition 2, $P^{*}$ is an intervals partition. Moreover, the number of undelegated intervals in $P^{*}$ is $K$. Therefore, when we raise $r$, we enable a larger number of delegated intervals without reducing the feasible number of undelegated intervals. This means that conditional on delegation, the Writer's expected utility increases, because each delegated interval becomes narrower. But this means that $\delta\left(c^{*}\right)$ cannot decrease with $r$.

Thus, as the Writer's language becomes richer, he chooses to increase the degree of vagueness. A priori, one could expect that having richer "linguistic resources" should lead the Writer to delegate less to the Interpreter. However, a richer language implies that the Writer can create a larger number of contradictions. More contradictions imply a finer effective partition, hence a smaller loss from delegating events to the Interpreter. Note that as long as $\delta\left(c^{*}\right)>0$, the number of contradictions remains the same, in accordance with Proposition 2.

Let us turn to comparative statics with respect to $K$. In the previous sub-section, we saw that when $r=1, \delta\left(c^{*}\right)$ decreases with $K$ - i.e., that a longer instructions list implies a lower equilibrium degree of vagueness. It turns out that this is not a general result. Note that if $r$ is very large, it is possible to construct an instructions list that induces an effective partition with $2^{K}$ cells, such that every cell in the partition corresponds to a different element in the power set of $K$.

Using the same kind of reasoning as in Proposition 3, it can be shown that $P^{*}$ contains $K$ undelegated intervals of measure $\left(1-\delta\left(c^{*}\right)\right) / K$ each, as well as $2^{K}-K$ delegated intervals of measure $\delta\left(c^{*}\right) /\left(2^{K}-K\right)$ each. The implication of this feature of $P^{*}$ is that the number of delegated intervals increases almost exponentially with the length of the instructions list (as long as $r$ is sufficiently large). Thus, an increase in $K$ allows a huge refinement of the Interpreter's strategy, and therefore may raise the Writer's incentive to delegate. 
The following example illustrates how an increase in $K$ can result in an increase in the equilibrium degree of vagueness. Let $m=\frac{1}{15}$ and $r=3$, and compare the cases of $K=2$ and $K=3$. In both cases, $r$ is sufficiently large so that $P^{*}$ consists of $2^{K}$ intervals. When $K=2, \delta\left(c^{*}\right)=0.4644$, by Proposition 2. When $K=3$, given a degree of vagueness $\delta$, the Writer's expected utility conditional on a delegated interval is $-\frac{1}{225}-\frac{1}{300} \delta^{2}$, and his expected utility conditional on an undelegated interval is $-\frac{1}{108}(1-\delta)^{2}$. It follows that $\delta\left(c^{*}\right)=0.5$.

Although we cannot produce a closed solution for $\delta\left(c^{*}\right)$, we are able to provide an upper bound. Note that for any $(K, r)$ and any instructions list $c$, the Writer's expected utility conditional on a delegated interval is bounded from above by $-m^{2}$. This is a consequence of Proposition 1 . The degree of vagueness that the Writer would choose if this bound could be attained cannot be lower than the equilibrium degree of vagueness. The Writer's expected utility, given $\delta$ and conditional on an undelegated interval, is $-(1-\delta)^{2} / 12 K^{2}$. Therefore, the obtain an upper bound on $\delta\left(c^{*}\right)$, we need to solve the maximization problem

$$
\max _{\delta} \delta \cdot\left(-m^{2}\right)+(1-\delta) \cdot \frac{-(1-\delta)^{2}}{12 K^{2}}
$$

which yields

$$
\delta^{*}=\max (1-2 K m, 0)
$$

It follows that $\max (1-2 K m, 0)$ is an upper bound $\delta\left(c^{*}\right)$ for any $(K, r)$. This observation has the following implication.

Proposition 6 For any $r, \delta\left(c^{*}\right)=0$ if $K m>\frac{1}{2}$.

Thus, if $K$ is sufficiently large relative to the conflict of interests, the Writer will not delegate anything to the Interpreter, regardless of $r$. 


\subsection{Which type of complexity is more valuable?}

Our model relies on two notions of complexity: the number of instructions $K$, and the maximal number $r$ of elementary events on which every instruction can condition. If one viewed the length of the instructions list (in terms of the number of words that it contains) as an indicator of its writing complexity, then $K r$ would be a reasonable measure. The question naturally arises, suppose that the Writer were able to manipulate $K$ and $r$ while keeping $K r$ fixed, what would he choose to do? Would he prefer a long list of simple instructions, or a short list of complex instructions? The following result provides an answer.

Proposition 7 For a fixed Kr, the Writer's subgame perfect equilibrium payoff attains a maximum when $r=1$.

Proof. Fix $K r$. Every event $e_{k}^{*} \in E^{*}$ is a union of $r$ disjoint intervals. If we relabel each of these intervals differently, then it is as if we have $K^{\prime}=K r$ and $r^{\prime}=1$. The effective partition we have constructed is feasible, yet not necessarily optimal, given $\left(K^{\prime}, r^{\prime}\right)$. Therefore, the Writer can only benefit when switching from $(K, r)$ to $\left(K^{\prime}, r^{\prime}\right)$.

Thus, although both types of complexity are valuable to the Writer, the number of instructions $K$ is more valuable than the complexity $r$ of every individual instruction.

\section{Alternative constraints on interpretations}

The rationale for contradictory instructions in this model is that another agent will interpret them. Contradictions have an informational content that enables the two agents to transgress the limits on the instructions list's complexity. In this section we explore alternative constraints on the way the 
Interpreter interprets contradictions, which capture alternative "methods of interpretation". We shall see that the rationale for contradictions disappears under these alternative constraints.

We wish to emphasize that our focus in this paper on a "method of interpretation" which is bound by a "precedent system" (as well as to the written law or contract, in case there are no contradictions or lacunae) is not because we believe that it is more realistic or important than other methods, but because unlike the others we have examined, it generates contradictions in equilibrium. See Shavell (2006) and Posner (2005) for further discussion of various constraints on the interpretation of contracts.

\subsection{Maximal discretion}

Let us relax the constraint that $I$ is a measurable function of $P^{c}$. Instead, suppose that the Interpreter's strategy is a function $I:[0,1) \rightarrow \mathbb{R}$ that assigns actions to states. We impose one constraint on this function: if $a_{k}^{c}=a$ for every $e_{k}^{c} \in E^{c}$ for which $x \in e_{k}^{c}$, then $I(x)=a$. That is, the Interpreter cannot overrule an unambiguous instruction, but he has total freedom in any other case. This variant of the model fits situations in which courts are not bound by precedents.

The rationale for contradictions disappears in this case. All that the Writer needs to decide upon is the degree of vagueness $\delta(c)$. Given the lack of any constraint on the Interpreter's discretion in case of vague instructions, there is no need to distinguish between lacunae and contradictions: any state over which the Interpreter has discretion will induce the outcome $x+a=m$ with probability one. The Writer can attain the optimal degree of vagueness with a lacuna, and without introducing any contradiction. Although the equilibrium list can contain contradictions, there is no special reason for them, and the list may contain only a lacuna.

The optimal degree of vagueness in this case is given by (1). When the Interpreter has unlimited discretion in case of a lacuna, the Writer's 
expected utility conditional on delegation is precisely $-m^{2}$. The reason is that delegating an event to the Interpreter completely eliminates the variance of $x+a$ (conditional on this event). As we saw in Section 3.2, this leads to expression (1), which is larger than the equilibrium degree of vagueness in our original model.

Note that the outcome of this alternative model Pareto-dominates the outcome given by the original model. This exposes a weakness of the legislaturecourt interpretation. Recall that we interpret the assumption that $I$ is measurable with respect to $P^{c}$ as reflecting a precedent system. But our analysis in this sub-section reveals that such a system is Pareto-inferior to a system without precedents. The question arises, what is the rationale for the precedent system in the first place? In order to address this question, one would have to enlarge the scope of the model and incorporate the costs of interpreting contradictions and lacunae.

\subsection{Minimal discretion}

An alternative assumption, at the other extreme, is that when the Interpreter faces contradictory instructions, he can only pick one of the actions specified by the instructions. Formally, suppose that $I:[0,1) \rightarrow \mathbb{R}$ is a function from states to actions, but assume that whenever $J^{c}(x) \neq \varnothing, I(x) \in\{a \in \mathbb{R} \mid a=$ $a_{k}^{c}$ and $x \in e_{k}^{c}$ for some $\left.k\right\}$.

In this case, it is straightforward to see that there is no rationale for contradictions. Suppose that $x \in e_{k}^{c}, e_{j}^{c}$. The Interpreter is forced to choose between $a_{k}^{c}$ and $a_{j}^{c}$. Clearly, he will choose the action that is more favorable to him, which may be less favorable for the Writer. Therefore, the instructions list induces an effective partition which prescribes no more than $K$ different actions (excluding the actions induced by a lacuna). If the Writer avoided contradictions, he would be able to induce an effective partition which prescribes $K$ different actions (excluding the actions induced by a lacuna), but these actions are better for him than the ones chosen by the Interpreter. 


\section{Conclusion}

Our objective in this paper was modest: to construct the simplest possible model that is capable of accommodating contradictions in contracts, and to provide a complexity-based rationale for contradictions. The basic idea is that when writing complex contracts is costly, there may be an incentive to introduce contradictions, and leave for another agent the task of interpreting them. The effect of complexity on the measure of delegated states is sensitive to the complexity notion, as well as to the norms that constrain the interpretation of contradictions. Hopefully, these ideas may inspire further research on contract-theoretic models, in which the court actively interprets laws and contracts.

\section{References}

[1] Anderlini, L. and L. Felli (1994), "Incomplete Written Contracts: Undescribable States of Nature", Quarterly Journal of Economics 109, $1085-1124$.

[2] Anderlini, L. and L. Felli (1999), "Incomplete Contracts and Complexity Costs", Theory and Decision 46, 23-50.

[3] Anderlini, L., L. Felli and A. Postlewaite (2001), "Courts of Law and Unforeseen Contingencies", Institute for Law \& Economics Research Paper 01-05, University of Pennsylvania.

[4] Anderlini, L., L. Felli and A. Postlewaite (2003), "Should Courts Always Enforce What Contracting Parties Write?", STICERD Theoretical Economics discussion paper No. TE/03/464, LSE.

[5] Battigalli, P. and G. Maggi (2002), "Rigidity, Discretion and the Costs of Writing Contracts", American Economic Review 92, 798-817. 
[6] Crawford, V. and J. Sobel (1982), "Strategic Information Transmission", Econometrica 50, 1431-1451.

[7] Dye, R. (1985), "Costly Contract Contingencies", International Economic Review 26, 233-250.

[8] Farnsworth, E. (1999), Contracts, third edition. New York: Aspen.

[9] Lipman, B. (2003), "Language and Economics", N. Dimitri, M. Basili, and I. Gilboa, eds., Cognitive Processes and Rationality in Economics, London: Routledge.

[10] Posner, R. (2004), "The Law and Economics of Contract Interpretation", Texas Law Review 83, 1581.

[11] Shavell, S. (2006), "On the Writing and the Interpretation of Contracts", Journal of Law, Economics and Organization 22, 289-314. 